\title{
Reflets
}

Revue d'intervention sociale et communautaire

\section{Formation en intervention interculturelle dans un centre de réadaptation en déficience intellectuelle et trouble envahissant du développement}

\section{Geneviève Saulnier}

Volume 17, numéro 2, automne 2011

Représentations, pratiques et expérience dans le champ de santé : tendre des ponts entre des disciplines

URI : https://id.erudit.org/iderudit/1012132ar

DOI : https://doi.org/10.7202/1012132ar

Aller au sommaire du numéro

Éditeur(s)

Reflets, Revue d'intervention sociale et communautaire

ISSN

1203-4576 (imprimé)

1712-8498 (numérique)

Découvrir la revue

Citer cet article

Saulnier, G. (2011). Formation en intervention interculturelle dans un centre de réadaptation en déficience intellectuelle et trouble envahissant du développement. Reflets, 17(2), 127-148. https://doi.org/10.7202/1012132ar
Résumé de l'article

Cet article fait suite à une expérimentation récente de formation continue offerte à des intervenants travaillant au Pavillon du Parc, un centre de réadaptation en déficience intellectuelle et trouble envahissant du développement (CRDITED). Cette expérimentation, qui consiste en une étape exploratoire d'un projet de recherche doctorale, s'inspire des fondements de l'approche interculturelle et des principes de l'apprentissage expérientiel. En revenant d'abord sur les principaux enjeux de l'intervention dans le champ de la santé et des services sociaux en contexte de diversité culturelle, cette contribution présentera le contexte institutionnel et social dans lequel s'est inscrite cette formation ainsi que les fondements à partir desquels elle a été construite. À partir des commentaires des intervenants lors de l'évaluation, la conclusion soulignera les forces et les limites de ce modèle de formation.
Tous droits réservés @ Reflets, Revue d’intervention sociale et communautaire, 2012
Ce document est protégé par la loi sur le droit d'auteur. L'utilisation des services d'Érudit (y compris la reproduction) est assujettie à sa politique d'utilisation que vous pouvez consulter en ligne. 


\section{Formation en intervention}

interculturelle dans un centre

de réadaptation en déficience intellectuelle et trouble envahissant du développement

\section{Geneviève Saulnier}

Étudiante au doctorat en service social, Université d'Ottawa

Agente de planification, de programmation et de recherche au Centre de réadaptation en déficience intellectuelle et trouble envahissant $d u$ développement (CRDITED), Pavillon du Parc ${ }^{1}$

Résumé

Cet article fait suite à une expérimentation récente de formation continue offerte à des intervenants travaillant au Pavillon du Parc, un centre de réadaptation en déficience intellectuelle et trouble envahissant du développement (CRDITED). Cette expérimentation, qui consiste en une étape exploratoire d'un projet de recherche doctorale, s'inspire des fondements de l'approche interculturelle et des principes de l'apprentissage expérientiel. En revenant d'abord sur les principaux enjeux de l'intervention dans le champ de la santé et des services sociaux en contexte de diversité culturelle, cette contribution présentera le contexte institutionnel et social dans lequel s'est inscrite cette formation ainsi que les fondements à partir desquels elle a été construite. À partir des commentaires des intervenants lors de 
l'évaluation, la conclusion soulignera les forces et les limites de ce modèle de formation.

Mots clés : intervention interculturelle, formation, déficience intellectuelle, trouble envahissant du développement, apprentissage expérientiel

\section{Abstract}

This paper present a recent experiment of intercultural training offered to practitioners at Le Pavillon du Parc, a readaptation center for people with an intellectual disability or autism spectrum disorder. This experiment consists in an exploratory stage of a doctoral research project and is based on intercultural approach and principles of experiential learning. This paper will describe and highlight the original model of training by presenting its theoretical basis and terms in the specific context of Le Pavillon du Parc. Finally, the perceptions of clinicians who were involved in this training activity will be presented in terms of their assessment of the strengths and limitations of this model.

Key words: intercultural intervention, intercultural training, intellectual disability, autism spectrum disorders, experiential learning.

\section{Introduction}

Cet article fait suite à une expérimentation récente de formation continue en intervention interculturelle offerte au Pavillon du Parc, un centre de réadaptation en déficience intellectuelle et trouble envahissant du développement (CRDITED) de la région de l'Outaouais. La mise en œuvre de cette formation répondait à un besoin énoncé par les intervenants eux-mêmes d'être mieux soutenus dans l'adaptation de leurs pratiques en contexte de diversité ethnique et culturelle.

Dans les pages qui suivent, à partir d'un ancrage particulier qui est celui de ma position de praticienne-chercheuse et de 
formatrice, je présenterai l'activité de soutien en intervention interculturelle mise en œuvre au Pavillon du Parc. Ici, l'expression "praticienne-chercheuse » renvoie non seulement à ma position de chercheuse menant sa recherche sur son terrain professionnel, mais souligne également l'articulation et l'imbrication de la recherche et de l'intervention dans le cadre de ce projet. La perspective sousjacente à la posture de praticien-chercheur étant de permettre à la pratique professionnelle de pénétrer l'activité de recherche dans le but de l'étayer et de façon dialogique, que l'activité de recherche oriente, voire renouvèle, la pratique professionnelle (De Lavergne, 2007).

Cette articulation entre des objectifs liés à la pratique et d'autres liés à la recherche a été en quelque sorte le moteur de ce projet. La pénétration de la recherche dans la pratique a permis de documenter non seulement le processus d'implantation de cette formation, mais aussi de permettre une meilleure définition des bases théoriques et pédagogiques qui l'ont animé et qui en font son originalité. Parallèlement, la conduite de cette formation et l'interpénétration "recherche-pratique " m'ont aussi permis de réaliser une phase préliminaire et exploratoire de mon projet de thèse et plus précisément de construire l'objet de ma recherche doctorale. Celle-ci s'inscrit dans le renouvèlement et l'adaptation des pratiques d'intervention en déficience intellectuelle et en trouble envahissant du développement en contexte de diversité socioculturelle.

Tout au long de cet article, je tenterai de mettre en évidence les éléments qui ont inspiré l'approche de formation en intervention interculturelle développée au Pavillon du Parc, en revenant d'abord sur les principaux enjeux de l'intervention dans le champ de la santé et des services sociaux en contexte de diversité culturelle. Puis, je présenterai le contexte institutionnel et social dans lequel s'est inscrite cette formation ainsi que les fondements à partir desquels elle a été construite. En m'appuyant enfin sur les commentaires des intervenants ayant participé à la formation, je présenterai en guise de conclusion l'évaluation qu'ils en ont faite au regard de ses retombées, de ses forces et de ses limites. 


\section{La prise en compte de la diversité culturelle en santé et services sociaux}

"...les établissements

doivent tenir compte

des particularités

linguistiques et

culturelles pour

favoriser l'accessibilité

des services aux

personnes issues

des différentes

communautés

culturelles. »
La préoccupation des établissements de santé et de services sociaux du Québec à l'égard de l'adaptation des services, des programmes et des pratiques en contexte de diversité culturelle est une question récurrente depuis le milieu des années quatre-vingt (Chan-Yip, 1985; Bibeau, 1987; Chiasson-Lavoie, 1992; Cohen-Emerique, 1992). Du premier Comité pour l'implantation du plan d'accès à l'égalité à l'intention des communautés culturelles (CIPACC, 1981-1984) à la récente Commission Bouchard-Taylor (20072008) où de nombreux établissements ont fait valoir leurs points de vue, toute une série de mesures, de programmes et de politiques ont été proposés afin de rendre les services sociaux et de santé plus accessibles et mieux adaptés à la réalité des personnes immigrantes et réfugiées.

Dans les institutions québécoises de santé, la prise en compte de la diversité culturelle s'inscrit dans la Loi sur les services de santé et les services sociaux du Gouvernement du Québec (LSSS). L'article 2.5 de cette loi stipule que «les établissements doivent tenir compte des particularités linguistiques et culturelles pour favoriser l'accessibilité des services aux personnes issues des différentes communautés culturelles".

Malgré la volonté politique et institutionnelle d'accroitre l'accessibilité et l'adaptation des services, plusieurs recherches mettent à jour une distance entre les établissements de santé et les communautés culturelles. Du côté des établissements, cette distance se traduit par le défi d'adapter les services offerts non seulement au regard des attentes et des besoins des personnes immigrantes (Battaglini, 2010; Fortin, 2004; Legault, 1999; Heneman, et collab., 1994), mais aussi dans la prise en compte de la diversité des représentations de la santé, de la maladie, de la souffrance et des troubles vécus et ressentis (De Plaen, 2004; Moro, 2002; Massé, 1995). 
"...Le pronostic de guérison et de mieuxêtre s'améliore, sur les plans physiologiques et psychiques, quand on tient compte des variables humaines et sociales dans l'évaluation et le traitement. »
De l'avis de plusieurs chercheurs et intervenants, la prise en compte des dimensions linguistique, culturelle, religieuse et sociale des individus serait essentielle à la réussite de l'intervention (Battaglini, 2010; De Plaen, 2004; Fortin, 2004). À cet effet, différentes études ont montré l'importance de considérer ces variables dans l'évaluation diagnostique, les plans d'intervention et les traitements proposés (De Plaen, et collab., 2005; De Plaen, 2004; Ntetu et Vissandjée, 1997). Selon certains, cette prise en compte influerait sur les processus de guérison : «Le pronostic de guérison et de mieux-être s'améliore, sur les plans physiologiques et psychiques, quand on tient compte des variables humaines et sociales dans l'évaluation et le traitement » (Fortin, 2004, p. 65). Sur le plan de la pratique clinique, ces données viennent donc suggérer que pour être efficace sur le plan thérapeutique, l'intervention doit s'ouvrir au pluralisme et à l'altérité.

Cependant, intégrer ces éléments d'altérité à l'intérieur des perspectives biomédicales de nos systèmes de santé et de services sociaux comporte un certain nombre de défis pour l'intervention. Pour répondre à ces défis et préoccupations, différents modèles et approches ont été développés dans le but de renforcer la sensibilité interculturelle des intervenants et ainsi favoriser une pratique mieux adaptée au pluralisme et à la diversité. Au cœur de ces approches et de ces modèles se trouve l'intervention dite « interculturelle ».

\section{L'intervention interculturelle en santé et services sociaux : une approche de prise en compte de la diversité}

L'intervention interculturelle fait partie des approches d'intervention développées dans le but de prendre en compte les différentes dimensions de la diversité. Dès le début des années quatre-vingt, plusieurs chercheurs se sont penchés sur la notion d'intervention interculturelle afin d'en circonscrire les contours. 
"...l'intervention interculturelle met de l'avant une vision de l'altérité qui se décline en de nombreuses dimensions : âge, sexe, statut social, etc. "
Je m'attarderai ici à présenter le regard de quelques-uns des auteurs phares ayant contribué à développer cette approche.

Cohen-Emerique (1993), l'une des pionnières en la matière, décrit l'intervention interculturelle comme une approche qui se fonde sur le respect de la personne, de sa vision du monde, de son système de valeurs et de ses besoins. Selon cette auteure, le respect de la personne se traduit par le respect de ses identités socioculturelles " avec ses multiples facettes d'appartenance ethnique, nationale, régionale, religieuse, de classe sociale, dans une approche dynamique et non-essentialiste, c'est-à-dire une identité en évolution et en changement au cours des années de vie dans le pays d'accueil»(Cohen-Emerique, 1993, p. 71).

Abdallah-Pretceille (1989) définit l'interculturel comme une interaction entre deux identités qui se donnent mutuellement un sens et dans un contexte à redéfinir chaque fois. Elle ajoute que l'intervention interculturelle se pose comme une relation entre deux individus ayant intériorisé dans leur subjectivité une culture unique, en fonction de leur âge, de leur sexe, de leur statut social et de leur trajectoire personnelle.

Pour Camilleri (1989; 1995), l'intervention interculturelle évoque la prise en compte de la diversité sous toutes ses formes socialement significatives et dans laquelle la variable culturelle n'est qu'une dimension de l'altérité prise dans son ensemble.

Ainsi, l'intervention interculturelle met de l'avant une vision de l'altérité qui se décline en de nombreuses dimensions : âge, sexe, statut social, etc. À travers l'intervention interculturelle, la culture n'aurait donc pas préséance sur les autres variables et l'appartenance à de grands ensembles culturels ne serait pas suffisante pour comprendre les besoins, le vécu, l'expérience et la réalité des personnes immigrantes et réfugiées qui sollicitent des services sociaux et de santé ou qui en bénéficient (Bélanger, 2000).

Parallèlement à cette vision décloisonnée et multidimensionnelle des appartenances identitaires suggérée par les pionnières de l'intervention interculturelle, des travaux récents montrent cependant une réification de la culture et une surdétermination 
«Dans leurs interventions, les professionnels ont des questionnements fréquents par rapport à des attitudes, à des comportements et à des positionnements culturellement marqués...» du culturel dans l'appréhension des défis relatifs à l'intervention en contexte de diversité (Cognet, 2007). En d'autres mots, à travers l'intervention, la culture de "l'autre " semble apparaitre comme le principal défi à surmonter.

Ce constat se confirme dans différentes recherches portant sur l'expérience des intervenants travaillant en contexte de diversité culturelle. En effet, comme le soulignent Kanouté, et collab. (2007), les intervenants ont souvent l'impression d'un hiatus entre leurs propres cadres de référence culturelle et ceux des personnes auprès desquelles ils interviennent. Dans leurs interventions, les professionnels ont des questionnements fréquents par rapport à des attitudes, à des comportements et à des positionnements culturellement marqués; « la conception des rapports hommes/ femmes ou adultes/enfants, la représentation de la santé ou de la maladie, la compréhension des services offerts " (Kanouté, et collab., 2007, p. 247) figurent parmi les différences culturelles éventuellement perçues par les intervenants.

Sans reprendre tout le débat autour de l'usage inflationniste du concept de culture dans le champ de la santé et des services sociaux, rappelons à l'instar de Cognet (2007, p. 58) que la référence à la culture dans le champ de la santé comporte un certain nombre de risques:

La prise en compte de la culture dans la santé ne devrait jamais être décontextualisée, en l'isolant des conditions sociales, historiques et politiques qui la sous-tendent. Elle ne doit jamais faire oublier que les pratiques des usagers doivent s'appréhender dans le contexte des interactions avec des praticiens et un système de santé qui ne sont jamais neutres. Nous devons adopter avec la culture une posture réflexive et toujours nous demander pourquoi nous la convoquons dans la relation à l'autre au cours des interventions cliniques. [Bref, adopter une posture qui] annihile toute prétention à savoir mieux que l'autre ce qui est bon pour lui, pour sa santé, pour son bien- 
"...les intervenants

disent que

l'intervention

interculturelle

nécessite l'acquisition

de connaissances

et d'aptitudes

complémentaires à leur formation de base..." être, sans pour autant interdire d'interroger les différentes valeurs qui soutiennent ces principes et les nôtres tout comme les registres dans lesquels ils s'expriment.

En contexte de diversité culturelle, les conditions sociales, historiques et politiques sous-jacentes aux relations entre les professionnels et les usagers des services sociaux et de santé peuvent représenter des angles morts de l'intervention. La culture se pose souvent comme un puissant filtre et ce qui demeure à l'avant-plan est davantage retenu : les codes de communication qui different, les différences de valeurs et de normes relatives à la santé ou encore la référence au religieux pour expliquer des malaises physiques.

Les parcours migratoires, les conditions d'intégration des nouveaux arrivants, l'ambigüité de la relation et quelquefois de la communication avec les immigrants, la complexité des interventions et des problèmes parfois rencontrés qui peuvent être nouveaux, inconnus ou inédits, l'inconfort devant la différence et les risques éthiques et déontologiques de pratiques vues comme inhabituelles sont autant d'éléments qui amènent une spécificité à l'intervention en contexte de diversité socioculturelle.

Dans ce contexte, les professionnels en viennent parfois à douter de la pertinence de leurs connaissances et de la validité de leurs approches (Bélanger, 2000). Ils peuvent vivre de la confusion, une perte de repères et un sentiment d'impuissance impliquant souvent une mise à l'épreuve de leurs savoirs. "Placés dans des situations où une dimension interculturelle heurte de front leur savoir-faire professionnel, les intervenants s'interrogent sur leurs compétences » (Bilodeau, 1993, p. 26). À ce titre, les intervenants disent que l'intervention interculturelle nécessite l'acquisition de connaissances et d'aptitudes complémentaires à leur formation de base (Battaglini, 2010) 


\section{Favoriser la formation en intervention interculturelle des professionnels en santé et services sociaux}

"Pour répondre aux besoins des intervenants d'être soutenus et pour renforcer leurs capacités à adapter leurs interventions, un large éventail de formations en intervention interculturelle est disponible...»
Dans une enquête réalisée par Battaglini (2010) auprès de neuf Centres locaux de services communautaires (CLSC) de la région de Montréal, les intervenants interrogés ont exprimé que la spécificité de l'intervention interculturelle exigeait l'acquisition de connaissances, d'attitudes, de compétences et de stratégies particulières permettant d'offrir une réponse mieux adaptée aux besoins et aux réalités des clientèles immigrantes. Pour adapter leurs interventions et tendre vers une plus grande prise en compte de l'altérité dans leurs pratiques, ces intervenants disent avoir besoin d'être davantage soutenus et insistent sur la pertinence de participer à des activités de formation continue en intervention interculturelle.Aussi, dans cette même enquête, la presque totalité des intervenants interrogés (97\%) considérait qu'il devrait y avoir une plus grande offre de formations pour les intervenants œuvrant en santé et services sociaux en contexte de diversité.

Pour répondre aux besoins des intervenants d'être soutenus et pour renforcer leurs capacités à adapter leurs interventions, un large éventail de formations en intervention interculturelle est disponible et s'offre à travers le réseau québécois de la santé et des services sociaux.

Ces formations peuvent être de type "classique » et offertes par un expert, prendre la forme de conférences scientifiques ou encore de séminaires, voire de supervisions professionnelles auprès d'équipes multidisciplinaires (Battaglini, 2010). La plupart se présentent toutefois sous forme de sessions d'un ou de deux jours caractérisées par l'acquisition de connaissances sur diverses composantes culturelles et migratoires ayant pour objectif de permettre aux intervenants d'accroitre leurs compétences et leur sensibilité culturelle (Foulks, Westermeyer et Ta, 1998).

Toutefois, selon Rousseau, et collab. (1995), l'efficacité de ce type de formation n'a pas été établie. Il est en effet permis 
"...la formation

visant le

développement

d'une sensibilité

interculturelle et

l'adaptation des

pratiques en contexte de diversité devrait

s'attarder à la culture prise au sens large et à la façon dont l'individu utilise ses diverses appartenances..." de croire que le fait d'avoir reçu une formation spéciale sur les dimensions culturelles et migratoires n'influe pas nécessairement sur les pratiques.

Au regard des fondements de l'intervention interculturelle, la formation visant le développement d'une sensibilité interculturelle et l'adaptation des pratiques en contexte de diversité devrait s'attarder à la culture prise au sens large et à la façon dont l'individu utilise ses diverses appartenances - sociales, professionnelles, culturelles, linguistiques ou religieuses pour s'exprimer et se définir. Parallèlement, les participants à la formation devraient être amenés à interroger leur propre identité et leurs appartenances par rapport à celles d'autrui et ainsi expérimenter la décentration. «La formation [doit façonner] chez l'intervenant la compétence interculturelle qui consiste à confronter les univers de sens culturellement marqués, celui du client et le sien, pour aboutir à une intelligibilité coconstruite " (Kanouté, et collab., 2007, p. 246).

La formation en intervention interculturelle devrait également viser le respect des différences et la capacité de mieux comprendre non seulement la complexité des cultures, mais celle également de tout être humain. Elle devrait permettre de surmonter les fausses dichotomies, les antagonismes des paradoxes apparents ainsi que la résistance au changement, celui qui est vécu comme une menace identitaire. Par ailleurs, la formation interculturelle devrait éviter toute perspective essentialiste qui enferme les individus dans des catégories culturelles ou ethniques définies en dehors d'eux. Elle devrait de plus éviter comme approche de comprendre l'autre en excluant l'analyse de soi. Finalement, en plaçant les enjeux de l'altérité au centre de son questionnement, la formation interculturelle ne doit pas occulter les contextes et passer sous silence les aspects sociaux, politiques et économiques ainsi que les rapports de pouvoir qui transcendent les interventions (Cognet, 2007; Kanouté, et collab., 2007; Costanzo et Vignac, 2001, dans Kanouté, et collab., 2007). 


\section{La mise en œuvre d'une formation en intervention interculturelle dans un centre de réadaptation en déficience intellectuelle et en trouble envahissant du développement}

\section{Le Pavillon du Parc}

"...le constat des gestionnaires et des intervenants de même que les statistiques sociodémographiques de la région permettent d'avancer que depuis les dernières années, il $y$ a bel et bien une hausse significative de la clientèle issue de l'immigration et des communautés culturelles. »
Le Pavillon du Parc est un centre de réadaptation en déficience intellectuelle et en trouble envahissant du développement (CRDITED) de la région de l'Outaouais. En tant qu'établissement du réseau de la santé et des services sociaux, sa mission est d'offrir des services spécialisés d'adaptation et de réadaptation aux personnes présentant une déficience intellectuelle ou un trouble envahissant du développement. Parallèlement, il offre des services de soutien et d'accompagnement aux familles et aux proches de ces dernières ainsi qu'aux différents partenaires œuvrant de près ou de loin auprès d'elles.

Comme tous les établissements de santé et de services sociaux situés dans les grands centres urbains, le Pavillon du Parc est de plus en plus touché par le phénomène de la diversité culturelle. Bien que l'établissement ne possède pas d'outils statistiques et de monitorage lui permettant de mesurer la proportion de ses usagers appartenant à une communauté culturelle, le constat des gestionnaires et des intervenants de même que les statistiques sociodémographiques de la région permettent d'avancer que depuis les dernières années, il y a bel et bien une hausse significative de la clientèle issue de l'immigration et des communautés culturelles.

À ce chapitre, les statistiques démographiques de la population de la ville de Gatineau - la plus grande agglomération de la région de l'Outaouais - démontrent que pour la seule année 2010, cette ville a vu bondir de $28,11 \%$ son nombre de nouveaux résidents issus de l'immigration. Ainsi, avec ses 8,7\% de personnes nées à l'étranger établies sur son territoire, Gatineau est la deuxième ville 
en importance au Québec, après Montréal, pour le nombre de personnes immigrantes et réfugiées (Ville de Gatineau, 2011).

Il va sans dire que les intervenants du réseau de la santé et des services sociaux de la région de l'Outaouais sont de plus en plus touchés par les défis que pose l'intervention auprès de familles immigrantes et réfugiées. C'est donc dans ce contexte que les intervenants du Pavillon du Parc ont souhaité être soutenus sur le plan de l'intervention interculturelle.

\section{Modalités de la formation en intervention interculturelle au Pavillon du Parc}

L'activité de formation en intervention interculturelle s'est déroulée durant l'année 2011, sur une période de six mois, et elle a réuni à raison d'une fois par mois un groupe multidisciplinaire formé de quinze intervenants, tous animés par le désir de réfléchir ensemble sur leur pratique d'intervention en contexte de diversité culturelle.

La formation étant non obligatoire et l'inscription volontaire, on peut supposer que les intervenants s'y étant inscrits manifestaient déjà un certain intérêt et une préoccupation personnelle ou professionnelle face à la question de l'interculturel.

Les participants à l'activité de formation étaient issus de différentes disciplines, psychoéducation, orthophonie, ergothérapie, travail social, et présentaient différentes caractéristiques sociodémographiques. Le groupe était constitué d'hommes et de femmes, âgés de 20 à 50 ans; certains vivaient en milieu rural, d'autres en milieu urbain; certains venaient du Québec ou de l'Ontario et d'autres étaient originaires de l'Europe, de l'Afrique ou des Antilles. C'est donc dire que le groupe lui-même était diversifié sur le plan des appartenances professionnelles, sexuelles, générationnelles, géographiques et culturelles. 


\section{Un modèle de formation inspiré de l'approche réflexive et des fondements de l'apprentissage expérientiel}

Alors que les modèles administratifs dont s'inspirent de plus en plus les établissements de santé et de services sociaux proposent une formation axée sur l'acquisition de compétences précises permettant une standardisation des pratiques (Rousseau, et collab., 2005), de plus en plus de chercheurs issus de différents milieux - éducation, santé, service social - insistent sur la pertinence de développer des formations dont l'approche est plus réflexive et qui se fondent sur le vécu et l'expérience des intervenants (Kanouté, et collab., 2007; De Plaen, 2005; Rousseau, et collab., 2005; Racine, 2000, Schön, 1994).

Ainsi, depuis les dernières décennies on assiste à l'émergence de travaux visant à réhabiliter la pratique comme lieu de production de savoirs (Rousseau, et collab., 2005; Racine, 2000; Réhaume, Sévigny et Tremblay, 2000; Schön, 1994). Ces travaux montrent que les praticiens ne font pas qu'adapter dans leurs pratiques les connaissances qu'ils ont acquises, mais qu'ils produisent des connaissances à même leurs réflexions, en cours d'action et sur l'action (Barbier, 1996; Schön, 1994).

Schön (1994), dont les travaux portent sur la réflexion en cours d'action, est reconnu pour avoir marqué le renouvèlement de la formation professionnelle en dynamisant le débat sur le statut des savoirs pratiques. En se penchant sur la notion de "pratique réflexive " et sur le rôle crucial de l'expérience dans l'apprentissage et dans la production de connaissances, l'auteur a tenté de redonner aux intervenants un statut d'acteur dans la construction de leur savoir.

Les activités de formation qui se fondent sur l'apprentissage expérientiel peuvent être de plusieurs ordres et emprunter des formes diverses. Toutefois, l'un des fondements essentiels de ce type de formation repose sur la remise en question de la 
"L'apprentissage expérientiel tend donc à une reconstruction de l'expérience à travers laquelle s'inscrit l'examen des connaissances et des pratiques. »

\section{"...la formation} en intervention interculturelle devrait aller au-delà des connaissances sur l'altérité pour permettre aux intervenants de se pencher sur leur propre cadre de référence. " transmission automatique de valeurs, de vérités et de connaissances qui ne cherchent pas à examiner leurs significations à la lumière d'expériences nouvelles et de nouveaux contextes. L'apprentissage expérientiel tend donc à une reconstruction de l'expérience à travers laquelle s'inscrit l'examen des connaissances et des pratiques. Ainsi, "le résultat du processus d'apprentissage expérientiel ne se limite pas à la création d'un nouveau savoir, mais peut inclure un changement d'attitude, une transformation de la conception de soi ou le développement de nouvelles habiletés " (Racine, 2000, p. 39).

Ces remises en question, ces changements d'attitudes et de conception de soi ainsi que le développement de nouvelles habiletés proposés par l'apprentissage expérientiel font écho aux fondements de l'intervention interculturelle et aux objectifs que devrait viser la mise en œuvre d'une formation dans ce contexte.

En effet, la formation en intervention interculturelle devrait aller au-delà des connaissances sur l'altérité pour permettre aux intervenants de se pencher sur leur propre cadre de référence. Cela, en relativisant les valeurs, les normes et les repères qui se posent souvent en absolu — et ils sont particulièrement nombreux dans le champ de la santé et des services sociaux - et ainsi, permettre de les mettre en contexte avec leurs dimensions politiques, économiques et institutionnelles.

Les fondements de l'apprentissage expérientiel ont inspiré la conduite de la formation en intervention interculturelle au Pavillon du Parc. La reconnaissance de l'expérience de chacun et l'échange de savoirs entre les participants ont été soutenus par une structure qui leur a permis de coconstruire à la fois le contenu et les objectifs de la formation. À cet effet, Theil (1989) distingue l'apprentissage expérientiel par celui qui se construit à partir d'objectifs établis par l'apprenant lui-même plutôt que par une source extérieure. 


\section{Coconstruction de la formation}

"...les intervenants ont été invités à nommer les forces à développer afin de mieux composer avec ces défis. »
Lors de la première rencontre, les participants ont été invités à identifier et à prioriser les principaux défis rencontrés à travers leurs expériences d'intervention en contexte de diversité culturelle. Parmi l'éventail des défis suggérés, cinq ont été retenus, chacun d'eux faisant l'objet d'une rencontre - bien entendu, sachant qu'il y aurait de nombreux croisements et recoupements :

1. La méconnaissance de la culture de «l'autre »;

2. La prise en compte des parcours migratoires;

3. La communication (barrière de langue, codes communicationnels, interprètes);

4. La diversité des représentations du handicap de développement;

5. Le manque de stratégies/habiletés/approches d'intervention interculturelle.

Dans la même foulée, les intervenants ont été invités à nommer les forces à développer afin de mieux composer avec ces défis. La notion de "forces » pouvait consister en des connaissances, des compétences, des qualités, des attitudes ou des habiletés. Huit forces à développer ont été proposées par les participants et sont devenues les objectifs de la formation :

1. Prendre conscience de ses préjugés;

2. Innover (être plus créatif) et utiliser de nouvelles stratégies d'intervention;

3. Développer ses connaissances sur les cultures;

4. Mieux comprendre les défis de l'immigration;

5. Suspendre son jugement, faire preuve de décentration et adopter une attitude d'ouverture;

6. Prendre conscience de la diversité des représentations du handicap de développement;

7. Reconnaitre son propre cadre de référence et d'interprétation de la réalité;

8. Éviter de poser des étiquettes culturelles pour expliquer les comportements. 
"L'apprentissage expérientiel a également été facilité par l'utilisation de matériel varié, tel que les discussions de cas, les extraits de films documentaires, les activités ludiques, les jeux interactifs et les mises en situation."

\section{"L'utilisation des} études de cas par le biais de discussions autour de vignettes cliniques a permis aux participants de relativiser et de confronter leur point de vue sur la pluralité des visions du monde..."
En tant que formatrice, j'ai proposé l'ajout de deux objectifs, soit la prise en compte des contextes socio-économiques et politiques dans l'intervention et la prise de conscience des enjeux de pouvoir dans l'intervention interculturelle.

Ce premier exercice de groupe a eu comme effet de mobiliser les intervenants et de les engager comme acteurs dans la formation. En participant à la coconstruction de la formation, tant sur le plan du contenu que sur celui des objectifs, les intervenants ont senti que cette formation leur appartenait. Cet engagement de leur part et cette mobilisation se sont traduits dans leurs commentaires d'appréciation tout au long de la formation et lors de l'évaluation finale.

L'apprentissage expérientiel a également été facilité par l'utilisation de matériel varié, tel que les discussions de cas, les extraits de films documentaires, les activités ludiques, les jeux interactifs et les mises en situation. Ce matériel de formation a non seulement permis l'intégration de nouvelles connaissances, mais a aussi suscité et provoqué les échanges, la discussion et même des débats entre les participants. Les discussions de cas, notamment, lesquelles se présentaient sous forme de vignettes cliniques, ont été utilisées à chacune des rencontres. À cet égard, les participants ont souligné l'apport d'une approche pédagogique permettant de faire des liens entre la théorie et la pratique et les sollicitant dans leurs expériences concrètes, du terrain.

Rousseau, et collab. (2005) ont expérimenté l'utilisation des discussions de cas dans le cadre de séminaires transculturels auprès d'intervenants en santé et services sociaux à Montréal. Selon ces auteurs, les discussions de cas provoquent la mise en évidence de perceptions différentes au sein d'un groupe et favorisent le développement de nouvelles façons de penser. Elles favoriseraient ainsi l'esprit critique, le développement d'une conscience de la relativité de toute connaissance, la mise à jour des présupposés ainsi qu'une meilleure prise en compte des contextes (Desser, Herzberg et Dirksen-Yoder, 2001, dans Rousseau, et collab., 2005).

L'utilisation des études de cas par le biais de discussions autour de vignettes cliniques a permis aux participants de relativiser et 
de confronter leur point de vue sur la pluralité des visions du monde, et tout particulièrement sur la diversité des représentations de la déficience intellectuelle ou du trouble envahissant du développement.

Enfin, les recherches portant sur l'apprentissage expérientiel ont également permis de mettre en lumière la variété des savoirs des intervenants : des savoirs cliniques formels, mais aussi des savoirs implicites, issus de leurs expériences personnelles et professionnelles (Rhéaume, Sévigny et Tremblay, 2000; Racine, 2000). Alors que ces savoirs ne sont pas toujours reconnus, ni par les intervenants eux-mêmes, ni par leurs milieux de travail, ces recherches indiquent que c'est dans la reconstruction de l'expérience que s'inscrit l'examen des pratiques et que cette reconstruction possède un potentiel de transformation des interventions et des habitudes instituées (Racine, 2000). À travers les échanges, notamment les discussions de cas, les participants ont pu exposer leurs différents savoirs, ce qui a favorisé chez eux une meilleure confiance en soi. Ce qui selon Geisinger et Carlson (1998) constitue une retombée importante de l'utilisation des discussions de cas.

\section{Conclusion}

"...une formation

en intervention

interculturelle s'avère

nécessaire selon eux, sinon essentielle, pour travailler dans le champ de la santé et des services sociaux."
Les intervenants ayant participé à la formation ont été unanimes quant à sa pertinence. Tenant compte des transformations sociales et culturelles de la région de l'Outaouais, une formation en intervention interculturelle s'avère nécessaire selon eux, sinon essentielle, pour travailler dans le champ de la santé et des services sociaux.

D'après les participants, la formule utilisée a favorisé et soutenu les apprentissages tout au long de la formation, qui a été qualifiée d'inédite et d'originale à la fois. Le fait de l'échelonner sur six mois à raison d'une rencontre aux quatre semaines a permis de nombreux aller-retour entre le terrain — lieu de pratique — et la formation - lieu d'échanges et de réflexion. La formule a permis 
"...les appréciations de la formation ont laissé entrevoir le besoin pour les intervenants de poursuivre leur réflexion sur l'intervention interculturelle. » d'intégrer les contenus, d'y réfléchir entre les rencontres et parfois même, de profiter des réunions dans les équipes respectives pour transmettre certains contenus et poursuivre les discussions. Les participants ont cependant constaté qu'une formation étalée sur six mois entrainait de l'absentéisme contrairement, selon eux, à une formation qui se donnerait en deux jours consécutifs. Les intervenants ont également insisté sur la stabilité du groupe en mentionnant qu'il s'agissait d'une condition incontournable, compte tenu de la nature de la formation basée sur la participation et l'implication de chacun et de la nécessité de développer un climat de confiance.

Par ailleurs, parmi les limites de ce modèle d'intervention, certains intervenants ont mentionné que leurs supérieurs étaient parfois réticents à les libérer étant donné l'étalement de la formation sur une période de six mois. Les cadres pouvaient avoir l'impression qu'il s'agissait d'une très longue formation, et ce, malgré le fait qu'en termes d'heures, la formation totalisait moins de trois journées complètes de travail. Il a donc été proposé que les gestionnaires soient mieux sensibilisés au modèle de formation et à l'approche préconisée, à ses avantages et à ses retombées, afin qu'ils en saisissent mieux la pertinence.

Enfin, les appréciations de la formation ont laissé entrevoir le besoin pour les intervenants de poursuivre leur réflexion sur l'intervention interculturelle. Les participants de cette cohorte ont recommandé que la formation soit offerte l'année prochaine à un autre groupe d'intervenants et proposé pour eux-mêmes la mise sur pied d'un groupe de pairs les réunissant une ou deux fois par année afin de poursuivre la réflexion sur l'intervention interculturelle.

Pour ma part, comme praticienne-chercheuse et doctorante en service social, cette expérimentation m'a permis de construire mon objet de recherche doctorale. Il m'appert que la volonté de rendre plus accessible les services sociaux et de santé aux personnes immigrantes passe inévitablement par l'adaptation des pratiques d'intervention. Mieux intervenir sur le plan de la diversité socioculturelle oblige les professionnels à adapter leurs pratiques en y greffant de nouvelles dimensions et variables, et 
ce, dans des contextes qui sont chaque fois à redéfinir. Mais dans le réseau de la santé et des services sociaux, cette adaptation des pratiques d'intervention en contexte de diversité socioculturelle semble à contre-courant de la tendance actuelle qui va vers leur standardisation. En réponse, le modèle de formation favorisant la réflexivité, l'échange, le va-et-vient entre la théorie et la pratique semble offrir aux intervenants un espace pour renégocier le sens de leurs pratiques, pour y réfléchir, pour les confronter parfois, peut-être aussi pour les remettre en question.

Toutefois, peut-on véritablement parler d'adaptation des pratiques sans connaitre les réalités des personnes immigrantes qui utilisent les services sociaux et de santé? L'adaptation des pratiques est-elle possible sans la compréhension de leur expérience de la maladie ou encore du handicap et sans prendre conscience de leur expérience à travers les services qu'elles reçoivent? Si plusieurs recherches ont porté sur différentes questions ou problématiques relatives à la santé des immigrants — santé mentale, périnatalité, sida, etc. - , peu d'entre elles se sont penchées sur la question de la déficience intellectuelle et du trouble envahissant du développement en contexte de diversité culturelle.

Enfin, l'expérimentation de cette formation en intervention interculturelle au Pavillon du Parc met en lumière toute l'importance de mieux connaitre les personnes et les familles immigrantes qui reçoivent des services en déficience intellectuelle ou en trouble envahissant du développement afin de mieux saisir la diversité de leur vécu. Cette phase préliminaire et exploratoire de ma recherche doctorale m'aura donc permis de dégager la pertinence de poursuivre mon projet sous trois axes différents, à savoir, 1. l'expérience des intervenants œuvrant en déficience intellectuelle ou en trouble envahissant du développement en contexte de diversité culturelle; 2.1 'expérience des familles issues de l'immigration vivant avec une personne ayant une déficience intellectuelle ou un trouble envahissant du développement; 3. et enfin, alimenté de la richesse des résultats des deux premiers axes, le soutien à l'adaptation des pratiques d'intervention dans le champ de la déficience intellectuelle et du trouble envahissant du développement en contexte de diversité culturelle. 


\section{Note}

$1 \quad$ Pour joindre l'auteure : genevievesaulnier@ssss.gouv.qc.ca; 819 770-1022 poste 201.

\section{Bibliographie}

ABDALLAH-PRETCEILLE, Martine (1989). «L'école face au défi pluraliste », dans Carmel Camilleri et Margalit Cohen-Emerique, Chocs de cultures : Concepts et enjeux pratiques de l'interculturel, Paris, L'Harmattan, p. 225-245.

ABDALLAH-PRETCEILLE, Martine (1997). " Pour une éducation à l'altérité ", Revue des sciences de l'éducation, Vol. 23, № 1, p. 123-132.

ALLIANCE DES COMMUNAUTÉS CULTURELLES POUR L'ÉGALITÉ DANS LA SANTÉ ET LES SERVICES SOCIAUX (2007). "Pour faire plus, il ne faut pas uniquement faire mieux, mais faire différemment ", Mémoire présenté aux consultations publiques sur les pratiques d'accommodements reliées aux différences culturelles, Commission Bouchard-Taylor, Montréal.

ASSOCIATION QUÉBÉCOISE D'ÉTABLISSEMENTS DE SANTÉ ET DE SERVICES SOCIAUX (2007). " Pour faciliter la prestation de soins et de services sans discrimination, de façon sécuritaire et personnalisée ", Mémoire présenté aux consultations publiques sur les pratiques d'accommodements reliées aux différences culturelles, Commission Bouchard-Taylor, Montréal.

BARBIER, Jean-Marie (1996). Savoirs théoriques et savoirs d'action, Paris, Presses universitaires de France, $305 \mathrm{p}$.

BATTAGLINI, Alex (2010). "Adapter, pas du tout, un peu, énormément, à la folie ", Montréal, Colloque sur les enjeux interculturels.

BATTAGLINI, Alex (2007). "Gestion et diversité culturelle : les services sociaux et de santé. Bibliographie thématique ", Montréal, CSSS de Bordeaux-Cartierville-Saint-Laurent.

BÉLANGER, Mario (2000). «L'intervention interculturelle. Une recherche de sens et un travail de sens ", Service social, Vol. 49, № 1, p. 70-93.

BIBEAU, Gilles (1987). À la fois d'ici et d'ailleurs : les communautés culturelles du Québec dans leur rapport aux services sociaux et aux services de santé, Montréal, Les Publications du Québec, 213 p.

BILODEAU, Guy (1993). "Méthodologie de l'intervention sociale et interculturalité », Service social, Vol.42, No 1, p. 25-48.

CAMILLERI, Carmel (1995). "Relations et apprentissages interculturels : réflexion d'ensemble ", dans Martine Abdallah-Pretceille et Alexander Thomas (dirs.), Relations et apprentissages interculturels, Paris, Armand Collin, p. 135-144.

CAMILLERI, Carmel (1989). "La culture et l'identité culturelle : champ notionnel et devenir ", dans Carmel Camilleri et Margalit Cohen-Emerique (dir.), Chocs de culture : concepts et enjeux pratiques de l'interculturel, Paris, L'Harmattan, p. 21-76.

CHIASSON-LAVOIE, Michèle (1992). L'approche interculturelle auprès des réfugiés et de nouveaux immigrants, Montréal, Centre des services sociaux du Montréal métropolitain, 77 p. 
CENTRE DE SANTÉ ET DE SERVICES SOCIAUX DE LA MONTAGNE (2007) «L'adaptation reliée à la gestion de la diversité dans le secteur de la santé et des services sociaux de la région de Montréal ", Mémoire présenté aux consultations publiques sur les pratiques d'accommodements reliées aux différences culturelles, Commission Bouchard-Taylor, Montréal.

CHAN-YIP, Alice M., et Michael S. KRAMER (1985). « Promotion of breast-feeding in a Chinese community in Montreal ", Canadian Medical Association Journal,Vol. 129, No 9, p. 955-958.

COGNET, Marguerite, et Catherine MONTGOMERY (2007). Éthique de l'altérité. La question de la culture dans le champ de la santé et des services sociaux, Québec, Presses de l'Université Laval., $261 \mathrm{p}$.

COHEN-EMERIQUE, Margalit (1993). "L'approche interculturelle dans le processus d'aide ", Santé mentale au Québec, Vol. 18, No 1, p. 71-91.

CONSEIL DE L'EUROPE (2011). Pour construire une culture institutionnelle inclusive. Compétences interculturelles dans les services sociaux, Strasbourg, Éditions du Conseil de l'Europe, $137 \mathrm{p}$.

DE LAVER GNE, Catherine (2007). «La posture du praticien-chercheur : un analyseur de l'évolution de la recherche qualitative ", Recherches qualitatives, Hors série, $N^{\circ} 3$, Actes du $1^{\text {er }}$ colloque international francophone sur les méthodes qualitatives.

DE PLAEN, Sylvaine (dir.) (2004). Soins aux enfants et pluralisme culturel, Montréal, Éditions de l'hôpital Sainte-Justine, 144 p.

DE PLAEN, Sylvaine, et collab. (2005). " Mieux travailler en situations cliniques complexes : l'expérience des séminaires transculturels interinstitutionnels ", Santé mentale au Québec, Vol. 30, No 2, p. 281-299.

DUCHARME, Francine, et collab. (2009). «La pratique dans un contexte pluriethnique : démarche en vue de la création d'une approche de négociation entre le personnel des services de soutien à domicile et les proches-aidantes d'un parent âgé ", Nouvelles pratiques sociales, Vol. 21, № 2, p. 137-156.

FORTIN, Sylvie, et Josiane LE GALL (2007). «Néonatalité et constitution des savoirs en contexte migratoire : familles et service de santé. Enjeux théoriques, perspectives anthropologiques ", Enfances, Familles, Générations, $N^{\circ}$ 6, p. 1-21.

FOULKS, Edward, Joseph WESTERMEYER et Karen TA (1998). "Developing curricula for transcultural mental health for trainees and trainers ", dans Samuel O. Okpaku, Clinical methods in transcultural psychiatry, Washington, American Psychiatric Press, p. 339-362.

GEISINGER, Kurt F., et Janet F.CARLSON (1998). "Training Psychologists ", dans Jonathan Sandoval, et collab., Test interpretation and diversity : achieving equity in assessment, Washington, DC, American Psychological Association, p. 377-386.

HENEMAN, Bernard, et collab. (1994). Adéquation des services aux jeunes familles immigrantes, Montréal, Direction de la santé publique, Régie régionale de la santé et des services sociaux de MontréalCentre.

KANOUTÉ, Fasal, et collab. (2007). «Les mots pour le dire et pour intervenir », dans Marguerite Cognet et Catherine Montgomery (dirs.) Éthique de l'altérité. La question de la culture dans le champ de la santé et des services sociaux, Québec, Presses de l'Université Laval, p. 241-26.

LANDRY, Francine (1989). "La formation expérientielle : origines, définitions et tendances ", Éducation permanente, Vol. 100/101, p. 13-21. 
LEGAULT, Gisèle, et Lilyane RACHÉDI (dirs.) (1999). L'intervention interculturelle, Boucherville, Gaëtan Morin Éditeur, 335 p.

LEGAULT, Gisèle, et Myriam LAFRENIÈRE (1992). « Situations d'incompréhensions interculturelles dans les services sociaux ", Santé mentale au Québec, Vol. 17, № 2, p. 113-131.

MASSÉ, Raymond (1995). Culture et santé publique, Montréal, Gaëtan Morin Éditeur, 499 p.

MARIN IZQUIERDO, Susanna (2007). Quelles formations pour développer les compétences interculturelles des intervenants sociaux en Wallonie?, Liège, Institut de Recherche, de Formation et Action sur les Migrations.

MORO, Marie-Rose (2002). Enfants d'ici venus d'ailleurs. Naître et grandir en France, Paris, La Découverte, $191 \mathrm{p}$.

MINISTÈRE DE LA SANTÉ ET DES SERVICES SOCIAUX (2011). «Loi sur les services de santé et les services sociaux, L.R.Q., c. S-4.2 ", Québec, Publication Québec.

MVILONGO-TSALA, Anselme (1995). "Formation et intervention interculturelle : défi pour l'Ontario français », Reflets, Vol. 1, № 1, p. 122-140.

NTETU,A. Lutumba, et BilkisVISSANDJÉE (1997). «Interactions en contexte clinique pluriculturel : regard critique et illustration ", dans Nous et les autres : les constructions identitaires au Québec et ailleurs, Québec, Turgeon, L., éd., CELAT, Presses de l’Université du Québec.

PINEAU, Gaston (1989). "La formation expérientielle en auto-, éco et co-formation ", Éducation permanente, 100-101, p. 67-77.

RACINE, Guylaine (2000). "La constitution des savoirs d'expérience chez des intervenantes d'organismes communautaires pour femmes sans-abri : un processus participatif, collectif et non planifié ", Nouvelles pratiques sociales, Vol. 13, No 1, p. 69-84.

RACINE, Guylaine (2000). La production de savoirs d'expérience chez les intervenants sociaux, Montréal, L'Harmattan, $170 \mathrm{p}$.

RHÉAUME, Jacques, Robert SÉVIGNY et Louise TREMBLAY (2000). La sociologie implicite des intervenants en contexte pluriethnique, № 6, Montréal, CLSC Côte-des-Neiges.

ROUSSEAU, Cécile, Michel PERRAULT et Pierre LEICHNER (1995). «Residents' perceptions of transcultural psychiatric pratice ", Community mental health Journal, Vol. 22, p. 517-531.

ROUSSEAU, Cécile, et collab. (2005). "Repenser la formation continue dans le réseau de la santé et des services sociaux. L'expérience des séminaires interinstitutionnels en intervention transculturelle ", Nouvelles pratiques sociales, Vol. 17, № 2, p. 109-125.

RUFAGARI, Marie-Claire (2010). "Le succès des interventions auprès des nouveaux immigrants passe inévitablement par l'adaptation des pratiques ", Montréal, Colloque sur les enjeux interculturels.

SCHÖN, Donald Alan (1994). Le praticien réflexif, Montréal, Éditions Logiques, 418 p.

THEIL, Jean-Pierre (1989). "L'autodidaxie comme type d'apprentissage ", Éducation permanente, Vol. 100/101, p. 31-38.

VILLE DE GATINEAU (2011). Profil démographique et socio-économique de la population immigrante dans la ville de Gatineau,réf. du 11 mai 2012,http://www.gatineau.ca/docs/histoire_cartes_statistiques/ immigration_diversite_culturelle/profil_demographique_socio_economique_population_ immigrante_ville_gatineau.fr-CA.pdf 